\title{
Collapse and fragmentation of Gaussian barotropic protostellar clouds
}

\author{
F. Gómez-Ramírez ${ }^{\dagger \ddagger}$, J. Klapp ${ }^{\ddagger+}$, Jorge L. Cervantes-Cota ${ }^{\ddagger *}$, G. Arreaga-García**, \\ D. Bahena ${ }^{\S}$ \\ ${ }^{\dagger}$ Facultad de Ciencias, Universidad Autónoma del Estado de México, El Cerrillo \\ Piedras Blancas, C.P. 5200, Estado de México, México. \\ $¥$ Instituto Nacional de Investigaciones Nucleares, Carretera México-Toluca S/N \\ La Marquesa, Ocoyoacac, C.P. 52750, Edo. de México. \\ * Berkeley Center for Cosmological Physics, LBNL, Berkeley, California 94720, \\ USA. \\ ** Centro de Investigación en Física de la Universidad de Sonora, A. P. 14740, \\ Hermosillo, C.P. 83000, Sonora, México. \\ $\S$ Astronomical Institute of the Academic sciences, Bočmí II 1401, 14130 Praha 4, \\ Czech Republic. \\ + jaime.klapp@inin.gob.mx
}

\begin{abstract}
We examine the problem of the collapse and fragmentation of molecular clouds with a Gaussian density distribution with high resolution, double precision numerical simulations using the GADGET- 2 code. To describe the thermodynamic properties of the cloud during the collapse -to mimic the rise of temperature predicted by radiative transfer- we use a barotropic equation of state that introduces a critical density to separate the isothermal and adiabatic regimes. We discuss the effects of this critical density in the formation of multiple systems. We confirm the tendency found for Plummer and Gaussian models that if the collapse changes from isothermal to adiabatic at earlier times that occurs for the models with a lower critical density, the collapse is slowed down, and this enhances the fragments' change to survive. However, this effect happens up to a threshold density below which single systems tend to form. On the other hand, by setting a bigger initial perturbation amplitude, the collapse is faster and in some cases a final single object is formed.
\end{abstract}

\section{Introduction}

The protostellar objects that begin their main sequence and pre-main sequence are mainly distributed in binary and multiple systems that suggests they were formed during the process of collapse and fragmentation of molecular clouds with dense cores and gas envelopes, see Sigalotti (2001a); Tohline (2002) and references therein. In recent years several authors have considered different realizations of molecular clouds to study their collapse and fragmentation. Although most 
fragmentation calculations apply to initially uniform conditions, see for instance Bodenheimer (2000), it is clear from the observations that molecular cloud cores are centrally condensed Ward-Thompson (2002); André (1998); Motte (1998). Thus, a number of collapse models starting from centrally condensed, Gaussian density profiles have also been made. A particular computationally demanding isothermal, Gaussian cloud model was first calculated in Boss (1991), and thereafter recalculated by other authors as a further test case to check both the likelihood of fragmentation during the isothermal collapse phase and the reliability of the numerical code results Burkert (1996); Truelove (1997); Boss (1998, 2000); Sigalotti (2001b); Sigalotti (2001c).

So far the great majority of this research has concentrated upon the early phases of star formation, when the collapse is dynamical, first isothermal and then nonisothermal. However, precise knowledge of the dependence of temperature on density at the transition from isothermal to nonisothermal collapse requires solving the radiative transfer problem coupled to a fully self-consistent energy equation. However, the full non-isothermal computation represents a severe computational burden imposed by solving the radiative transfer equations at high spatial resolution, even in the Eddington approximation. Therefore, it has been common to use instead a simple barotropic equation of state Boss (2000) that clearly simplifies the computational problem and it turns out to be a good approximation for the dynamical collapse of the molecular clouds, see Arreaga-García (2007); Arreaga (2008). In the present work, a barotropic equation of state is assumed to simulate the transition from isothermal to adiabatic collapse. The motivation of this study is to investigate the sensitivity of fragmentation to the effects of thermal retardation by varying the value of the critical density at which nonisothermal heating is assumed to begin. In Arreaga-García (2007); Arreaga (2008) it is studied the evolution of a Gaussian density profile, and found that by diminishing the critical density it enhances the fragmentation. A similar result was found for Plummer models in Arreaga-García (2010).

The present work is a continuation of the analysis in Arreaga-García (2007); Arreaga (2008) in which we employ a Gaussian density profile and perform the same type of numerical computations but now using double precision in the GADGET-2 code. Particularly, we study the effect of varying the critical density of the barotropic equation of state in the collapse and fragmentation of the molecular protostar. We also analyze the effect to increasing the initial perturbation amplitude.

\section{Initial conditions and collapse models}

According to astronomical observations, the regions from which stars are formed consist basically of molecular hydrogen clouds at a temperature of $\sim 10 \mathrm{~K}$. Therefore, the ideal equation of state is a good approximation to account for the thermodynamics of the gas in these clouds. The cloud models are based on the standard isothermal test case, as in the variant considered in Burkert (1993). However, once 
gravity has produced a substantial contraction of the cloud, the opacity increases, the collapse changes from isothermal to adiabatic and the gas begins to heat. To include this rise in temperature into our calculations, we use the barotropic equation of state proposed in Boss (2000).

In order to correctly describe the non-isothermal regime, one needs to solve the radiative transfer problem coupled to the hydrodynamic equations, including a fully self-consistent energy equation to obtain a precise knowledge of the dependence of temperature on density. The implementation of radiative transfer has already been included in some mesh-based codes. In SPH, the incorporation of radiative transfer has in general not been very satisfactory, perhaps with the exception of reference Whitehouse (2006), in which they used the f-limited diffusion approximation to model the collapse of molecular cloud cores. These authors suggested that there are important differences in the temperature evolution of the cloud when radiative transfer is properly taken into account.

However, after comparing the results of the simulations performed by

Arreaga-García (2007); Arreaga (2008) with those of reference Whitehouse (2006) for the uniform density cloud, it is concluded that the barotropic equation of state in general behaves quite well and that we can capture the essential dynamical behavior of the collapse. The simulations in this work are consequently carried out using the following barotropic equation of state:

$$
p=c_{i s o}^{2} \rho+K \rho^{\gamma},
$$

where $\gamma$ is the adiabatic exponent in the opacity thick regime and $K$ is a constant set by $K=c_{i s o}^{2} \rho_{\text {crit }}^{1-\gamma}$, where $\rho_{\text {crit }}$ defines the critical density above which the collapse changes from isothermal to adiabatic, and for a molecular hydrogen gas the ratio of specific heats is $\gamma=5 / 3$, because we only consider translational degrees of freedom.

With the above prescriptions, the local sound speed becomes

$$
c=c_{\text {iso }}\left[1+\left(\frac{\rho}{\rho_{\text {crit }}}\right)^{\gamma-1}\right]^{1 / 2},
$$

so that $c \approx c_{\text {iso }}$ when $\rho \ll \rho_{\text {crit }}$ and $c \approx C_{a d}=\gamma^{1 / 2} c_{\text {iso }}$.

The molecular cloud collapse simulations in this work begin with initial conditions in accordance to the thermodynamic model proposed in Burkert (1993). Accordingly, the models start with a spherical cloud of mass $M=1 M_{\odot}$, radius $R=4.99 \times 10^{16} \mathrm{~cm} \sim 0.016 \mathrm{pc}$, and at a temperature $T=10 \mathrm{~K}$. The initial model is composed by an ideal gas with an average molecular weight $\mu \sim 3$. We have chosen the initial sound speed $c_{i s o}$ and angular velocity $\omega_{0}$ in such a way that for all models the initial ratio of thermal and rotational energies to gravitational energy are such that $\alpha=E_{\text {therm }} /\left|E_{\text {grav }}\right| \sim 0.26$ and $\beta=E_{\text {rot }} /\left|E_{\text {grav }}\right| \sim 0.16$. The gas isothermal sound speed is $c_{i s o} \sim 1.90 \times 10^{4} \mathrm{~cm} \mathrm{~s}^{-1}$, and the average free fall time is $5.10 \times 10^{11} \mathrm{~s}$. Additionally, we impose a small perturbation to the density profile of the following form:

$$
\rho=\rho_{0}[1+\operatorname{acos}(m \phi)]
$$


where $m$ is an integer number, $\phi$ the azimuthal angle around the $z$-axes, and $a$ is the perturbation amplitude.

The chosen density profile is Gaussian with the above-mentioned initial conditions, as in Arreaga-García (2007); Sigalotti (2001a):

$$
\rho(r)=\rho_{c} \exp \left[-\left(\frac{r}{b}\right)^{2}\right],
$$

where $\rho_{c}=1.7 \times 10-17 \mathrm{grcm}^{-3}$ is the initial central density and $b \approx 0.578 R$ is a length chosen such that the density is 20 times smaller there. On the other hand, solid-body rotation is assumed at the rate of $\omega_{0}=1.0 \times 10^{-12} \mathrm{~s}^{-1}$.

\section{Numerical methods}

The computations of this work were performed using the parallel code GADGET2, which is described in full in Springel (2005). The code is suitable for studying isolated, self-gravitating systems with high spatial resolution. The code is based on the tree-PM methods for computing the gravitational forces and on standard SPH methods for solving the 3D Euler hydrodynamics equations. For a review on the theory and applications of SPH we refer the reader to Monagan (2005).

In order to set up the initial particle distribution, we first define a Cartesian box with sides equal to twice a specified radius $R_{b} \gtrsim R=4.99 \times 10^{16} \mathrm{~cm}$, and with its geometrical center coinciding with the origin $(x=y=z=0)$ of a Cartesian coordinate system. The box is then subdivided into regular cubics cells of volume $\Delta^{3}=\Delta x \Delta y \Delta z$ each. The spherical cloud is then copied within the box by placing an SPH particle in the center of each cell at distances $d \leq R$ from the origin, so that the region outside the sphere is empty. A little amount of disorder is added to the regular distribution of particles by shifting each particles a distance $\Delta / 4$ from its cell-center location and along a specified direction, which is chosen randomly among the three Cartesian axes. We defined the mass of particle $i$ at location $\left(x_{i}, y_{i}, z_{i}\right)$ to be $m_{i}=$ $\rho\left(x_{i}, y_{i}, z_{i}\right) \Delta^{3}$, where

$$
\rho\left(x_{i}, y_{i}, z_{i}\right)=\rho_{c} \exp \left[-\frac{x_{i}^{2}+y_{i}^{2}+z_{i}^{2}}{b^{2}}\right] .
$$

Solid-body rotation about the $z$-axis is assumed in a counter clockwise sense by assigning to particle $i$ an initial velocity given by $v_{i}=\left(\omega_{0} x_{i},-\omega_{0} y_{i}, 0\right)$. Finaly, the bar mode density perturbation given by equation (3) applied by modifying the mass of particle $i$ according to $m_{i} \rightarrow m_{i}\left[1+a \cos \left(m \phi_{i}\right)\right]$, where $\phi_{i}$ denotes the azimuthal position of that particle. The computations were performed in the parallel cluster of the National Institute of Nuclear Research-Mexico, equipped with 28 AMD Quadcore (64 bits) Opteron Barcelona processors. 


\section{Results}

In this section we present the results obtained using the GADGET- 2 code of the collapse and fragmentation of Gaussian molecular clouds. The collapse of the Gaussian cloud first calculated in Boss (1991), later by other authors Burkert (1996); Truelove (1997); Boss (1998); Sigalotti (2001b); Sigalotti (2001c); Arreaga-García (2007) using high spatial resolution, and in the present work we perform the same computations as in Arreaga-García (2007), but adding double precision and using $10^{7} \mathrm{SPH}$ particles in each simulation.

We present four different cases for the barotropic collapse with the parameters as shown in table 1 The results are illustrated with iso-density contour plots for a slide at the equatorial plane of the cloud in figures 1, 4 . The bar located at the bottom of the plots shows the $\log _{10}$ density range at a time $t$ and normalized with the initial central density $\rho_{c}$. A color scale is then associated with the value of $\log _{10}$. For instance, the color scale uses yellow to indicate higher densities, blue for lower densities, and green and orange for intermediate densities.

The free fall time $t_{f f} \approx \sqrt{3 \pi /\left(32 G \rho_{c}\right)}$ sets a characteristic time scale for the collapse of protostellar clouds which is given in terms of the central density and it is the same for all models, see for instance Arreaga-García (2010).

Table 1 Gaussian collapse models. The model types are explained as follows: The letter $\mathrm{G}$ refers to Gaussian, the number 6 refers to ten millions particles, the letters A, B, and C distinguish among the different critical densities, and finally the last digit refers to the amplitude of the initial perturbation.

\begin{tabular}{clll}
\hline Model & $\rho_{\text {crit }}\left(\mathrm{g} \mathrm{cm}^{-3}\right)$ & Amplitude $a$ & Final outcome \\
\hline G6A1 & $5 \times 10^{-12}$ & 0.1 & Binary \\
G6B1 & $5 \times 10^{-14}$ & 0.1 & Quadruple \\
G6C1 & $5 \times 10^{-15}$ & 0.1 & Single \\
G6B5 & $5 \times 10^{-14}$ & 0.5 & Single \\
\hline
\end{tabular}

The aim of the present models is to explore, using double precision variables, the sensibility of fragmentation to both the effect of thermal retardation due to nonisothermal heating that is controlled by the critical density, and the effect to increase of perturbation amplitude.

The construction of the initial conditions ensures that the collapse initial stage is similar for all models considered here. The initial phase of the collapse proceeds in such a way that the material falls down to the rotation plane, while material near the central midplane undergoes a weak expansion perpendicular to the rotation axis, causing the formation of two overdense blobs from the initial $m=2$ perturbation seed. At about the end of the first free-fall time, the expansion stops and the middle region begins to collapse, the blobs fall toward the center and merge to form a prolate structure. By this time, the overall cloud has already been compressed into a flat disk with an inner bar that begins to rotate. 
We begin the discussion with models G6A1, G6B1, seen in figures 1 and 2 The model G6A1 collapses faster than the corresponding single precision model reported in Arreaga-García (2007), but the overall dynamics looks similar. We now proceed to decrease the critical density, as shown in model G6B1. We find however important differences in comparison to Arreaga-García (2007), which reports as an end product a binary system formed by a transient quadrupole system, but we obtain as a final product a stable quadrupole system. The fact that by diminishing the critical density the fragmentation enhances, found for Gaussian Arreaga-García (2007); Arreaga (2008) and Plummer models Arreaga-García (2010), seems to be reproduced for models G6A1 and G6B1. However, by diminishing even more the critical density in model $\mathrm{G} 6 \mathrm{C} 1$, see table 1 the collapse and fragmentation is slowed down and the fragmentation is less favored, as seen in figures 2 and 3 .

The model G6B5, shown in figure 4, has a bigger initial perturbation amplitude that provokes that systems collapse earlier to form a filamentary structure, which evolves to a transient binary system that later collapses in a single system. The final system ejects significant amount of gas out of the core. In comparison the same model but with a smaller amplitude, model G6B1, forms a stable quadrupole system.
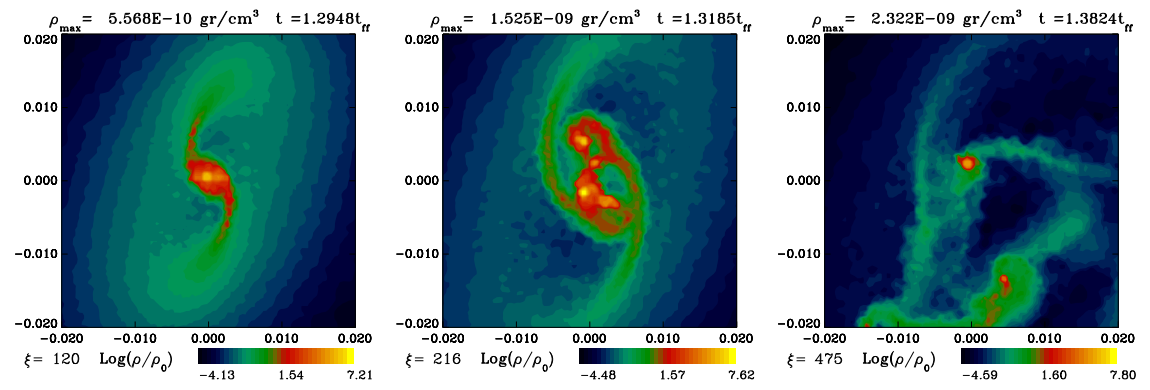

Fig. 1 Iso-density contour plots at the equatorial plane of model G6A1 for three different times.
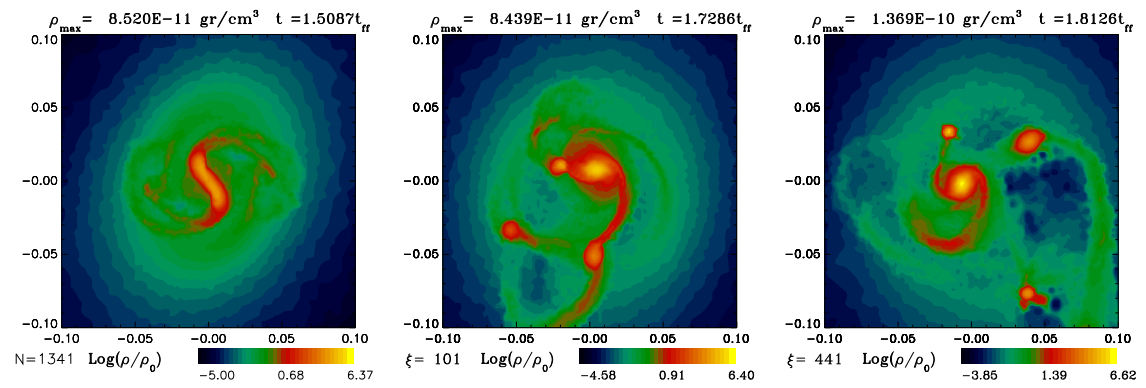

Fig. 2 Iso-density contour plots at the equatorial plane of model G6B1 for three different times. 

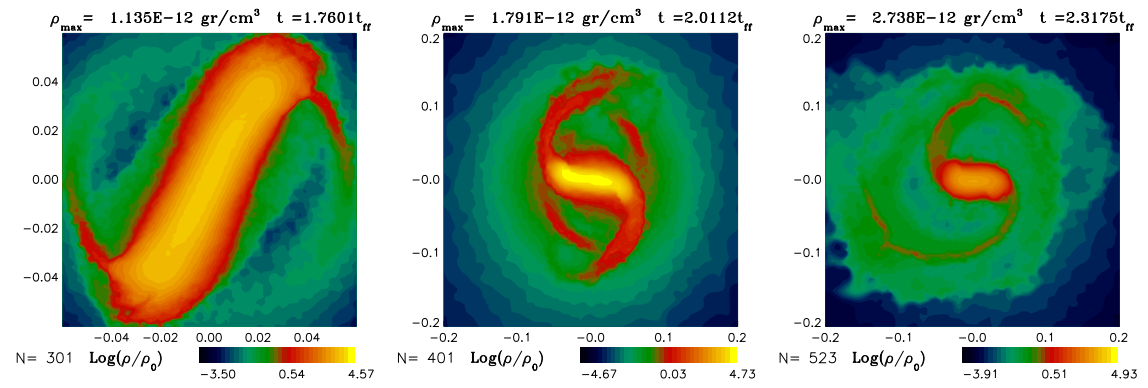

Fig. 3 Iso-density contour plots at the equatorial plane of model G6C1 for three different times.
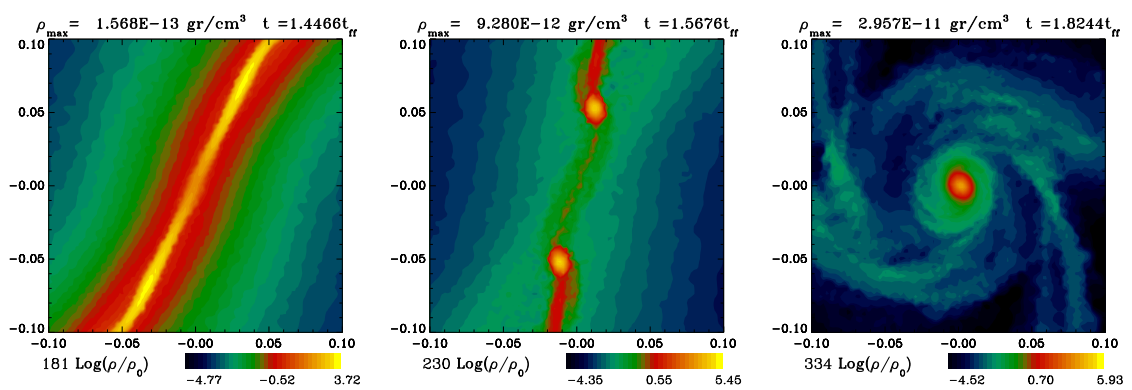

Fig. 4 Iso-density contour plots at the equatorial plane of model G6B5 for three different times.

\section{Conclusions}

In this work, we have followed the early phases of cloud collapse and fragmentation up to the formation of the proto stellar core using the GADGET-2 code with high spatial resolution and double precision, using $10^{7} \mathrm{SPH}$ particles. The initial conditions for the cloud models are chosen to be the standard isothermal test case, as in the variant considered in Burkert (1993), but for a centrally condensed, Gaussian cloud, that was first treated in Boss (1991), and further considered by other authors Arreaga-García (2007). The main results are summarized as follows:

By augmenting from single to double precision, the collapse happens earlier and the number of end products augments, as seen in our simulations in comparison the same models in Arreaga-García (2007).

On the other hand, we find that the effect of diminishing the critical density of the barotropic equation of state, provokes the collapse to slow down, and this enhances the fragments' change to survive. However, this effect happens up to a threshold density, as seen in our simulation G6C1, where considered a low critical density, $\rho_{c}=5 \times 10^{-15} \mathrm{~g} / \mathrm{cm}^{3}$, and a single system was formed.

Moreover, models with a bigger initial perturbation amplitude provoke that systems collapse earlier, and in some cases, in the form of a filamentary structure which evolves to a transient binary system that later collapses in a single system. 


\section{Acknowledgements}

J.L.C.C. thanks the Berkeley Center for Cosmological Physics for hospitality, and gratefully acknowledges support from a UC MEXUS-CONACYT Grant, and a CONACYT Grant No. 84133-F.

\section{References}

André, P., Bacmann, A., Motte, F., \& Ward-Thompson, D., in The Physics and Chemistry of the Interstellar Medium, ed. V. Ossenkopf, J. Stutzki, \& G. Winnewisser (Zermatt: GCA-Verlag), 241 (1998).

Arreaga-García G., Klapp J., Sigalotti, L. D. \& Gabbassov, R., Ap. J. 666, 290 (2007).

Arreaga, G., Saucedo, J., Duarte, R., Carmona, J., Rev. Mex. Astron. Astrofis. 44, 259 (2008).

Arreaga-García G., Klapp Escribano J. \& Gómez-Ramírez, F., A \& A 509, A96 (2010).

Bodenheimer, P., Burkert, A., Klein, R.I., Boss, A.P., in Protostars and Planets IV, Eds. V.G. Mannings, A.P. Boss and S.S. Russell, University of Arizona Press, Tucson (2000).

Boss, A. P., Nature, 351, 298 (1991).

Boss, A.P., Ap. J. 501, L77 (1998).

Boss, A.P., Fisher, R.T., Klein, R., McKee, C.F., Ap. J. 528, 325 (2000).

Burkert, A. \& Bodenheimer P., MNRAS, 264,798 (1993).

Burkert, A., Bodenheimer, P., MNRAS, 280, 1190 (1996).

Monagan, J.J., Rep. Prog. Physics 68, 1703 (2005).

Motte, F., André, P., \& Neri, R., A \& A 336, 150 (1998).

Sigalotti, L. D. \& Klapp, J., Intern. J. Mod. Phys. D 10, 115 (2001a).

Sigalotti, L. D. \& Klapp, J., A\&A 378, 165 (2001b).

Sigalotti, L. D. \& Klapp, J., in Exact Solutions and Scalar Field in Gravity: Recent Developments, ed. A. Macias, J. Cervantes, \& K. Laemmerzhal (Dordrecht: Kluwer), 223 (2001c).

Springel, V., MNRAS 364, 1105 (2005).

Tohline, J.E., Annu. Rev. of Astron. and Astrophys. 40, 349 (2002).

Truelove, J.K., Klein, R.I., McKee, C.F., Holliman, J.H., Howell, L.H., Greenough, J.A., Ap. J. 489, L179 (1997).

Ward-Thompson, D. Science, 295, 76 (2002).

Whitehouse, S.C., Bate, M.R., MNRAS 367, 32 (2006). 